\title{
Advancement of Bio inks in three Dimensional Bioprinting
}

\author{
Sumbul Ali $^{1}$ and Saima Majeed ${ }^{* 1,2}$ \\ ${ }^{1}$ Akhuwat Faisalabad Institute of Research, Pakistan \\ ${ }^{2}$ National Institute for Biotechnology and Genetic Engineering (NIBGE), Pakistan
}

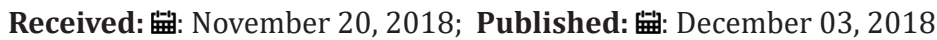

*Corresponding author: Saima Majeed, Akhuwat Faisalabad Institute of Research, Pakistan

\begin{abstract}
Three dimensional bioprinting is the modern technique extensively used in regenerative medicine and tissue engineering for the development of multifaceted biological structures such as complex tissues and organs. Computer aided design (CAD) is being used to design complicated three dimensional structures. After designing the desired structure, next step of bioprinting involves the deposition of "bio inks" in the designed shapes. These bio inks are enriched with living cells and any medium supporting and nourishing these cells. Various bioprinters and bio inks based on agarose, collagen, hyaluronic acid, fibrin, Matrigel and pluronic $\AA$ F-12 are discussed with respect to the advantages, disadvantages and suitability in the development of complex 3D biostructures. 3D bioprinting technology is a futuristic approach for tissue engineering and synthesis of other biological structures with greater feasibility which will help to overcome the hurdles in all medical fields.
\end{abstract}

Abbreviations: CAD: Computer Aided Design; EBB: Extrusion Based Bioprinter; LIFT: Laser Induced Forward Transfer; HA: Hyaluronic Acid

\section{Introduction}

Three dimensional bioprinting is the modern technique extensively used in regenerative medicine and tissue engineering for the development of multifaceted biological structures such as complex tissues and organs. An American inventor named Chuck Hull coined the concept of three dimensional printing in early 80's. Three dimensional bioprinting works by depositing cell-laden biomaterial in a predesigned architecture to engineer functional organs and tissues. Computer aided design (CAD) is being used to design complicated three dimensional structures by processing the data obtained from MRI, X-ray and other imaging techniques. In this way a more personalized, highly precise and patient specific structures could be created efficiently within minimum time and resources. After designing the desired structure, next step of bioprinting involves the deposition of "bio inks" in the designed shapes. These bio inks or biomaterials are enriched with living cells and medium supporting and nourishing these cells [1-2].

Rheological behavior, gelation kinetics, swelling properties and surface tension of bio inks are the key characteristics which influence printability. But the core aspect here is bio fabrication which mainly rely on bio ink dispensing. Thus it is necessary to choose appropriate dispensing technique for relevant bio ink. Three main methodologies vastly used for bioprinting includes; inkjet bioprinting, extrusion based bioprinting and laser assisted bioprinting [3]. Inkjet bioprinting is based on two techniques i.e. thermal inkjet bioprinting and piezoelectric bioprinting. Former vaporizes the bio ink using a heating element and creates pulses of pressure which expels the droplets out. While in piezoelectric bioprinting, acoustic waves are produced by piezoelectric crystals which forces the fluid out of the nozzle head. Droplet size of inkjet bioprinter is $10-50 \mu \mathrm{m}$ and its formation is mainly dependent on the surface tension which arises due to the cohesive forces in the molecules of the liquid. Inkjet bioprinters are vastly used for bioprinting owing to its precision and compatibility with various bio inks but on the other hand, nozzle blockage due to early gelation of bio ink and loss of viability of living cells due shear and heat stress reduces the efficiency of inkjet bioprinters [4].

Unlike inkjet bioprinter, extrusion based bioprinter (EBB) dispenses filaments of hydrogel which are 150-300 micrometer in diameter. Bio ink is fed to plastic syringes and it is extruded out via piston (pneumatic) or screw-driven (mechanical) assembly. Pneumatic bioprinting provides better control of flow rate of bio ink whereas mechanical assembly aids the spatial control and printing of viscous bio inks. Major drawback of this methodology is poor resolution and like inkjet bioprinting; compromised cell 
viabity and nozzle clogging also occurs [5-6]. Another methodology used for bioprinting is LIFT (Laser induced forward transfer) which is orifice free bioprinting technique. The assembly of LIFT consists of three layers i.e. donor substrate, an absorbing layer made up of gold or titanium and bio ink layer. Laser beam is focused on the absorbing plate which creates a pressure bubble due to local evaporation thus impelling a small quantity of hydrogel towards the collector platform by leaving the donor layer although a loose connection remains. This methodology addresses the nozzle clogging, shear and heat stress [7].

\section{Bio inks for 3D Bioprinting}

Raw material used for the manufacturing of 3D printed complex biological structures are considered as bio inks which the substances are mainly comprised of living viable cells or tissues and the matrix which holds these cells together and provide nutrition and support to the cells. Bio inks imitates extracellular matrix which allows adhering, proliferating and differentiating of bio printable cells. Selection of bio inks for 3D printing of the biomaterials is an imperative task. There is a pool of physical and chemical aspects of bio inks to be considered for bioprinting. Basic requirements for bio inks include bio printability, in situ gelation, viscoelasticity, biocompatibility with live cells, tissue regeneration, permeability of oxygen, nutrients and metabolic wastes, biodegradation and sheer thinning [8]. Recent studies have reported various biomaterials can be manipulated as bio inks. An overview of these materials will be discussed in following section. These biomaterials are categorized into two main classes; natural biomaterials and synthetic biomaterials. Both have their own distinct properties such as natural biomaterials show high degree of biomimicking and ability of self-assembling whereas stability, controllability and photo crosslinking are the key features of synthetic polymers.

\section{Agarose Based Bio inks}

Agarose is a polysaccharide which is derived from marine algae and has diverse application in biomedical field due its property of gel formation [9]. Agarose based bio inks imparts stability to the 3D structures and it can be used in conjunction with other biomaterials to increase the efficiency. A study has manipulated alginate, agarose and carboxymethyl-chitosan to develop functional neurons from neural cells derived from humans and printed the stable structures by encapsulating the cells [10]. A study has made a comparison between agarose and other hydrogels based on biocompatibility and printability by loading the gels with mesenchymal cells and 3D printed cartilage to examine the differentiation of cartilage to fibrocartilage. All the gels showed viability of around $80 \%$ while agarose and alginate showed higher cell differentiation $[11,12]$. Rapid gel formation, high degree of biocompatibility and rheological properties make it highly preferable for bioprinting. However, owing to its viscous nature it is not commonly used in inkjet bioprinters as it causes nozzle clogging [7].

\section{Collagen-Based Bio inks}

Collagen is a protein which is obtained naturally from the extra cellular matrix of many mammals and it is extensively used as bio ink for tissue regeneration, tumor modeling and other tissue engineering techniques. Owing to integrin-binding domains of collagen it facilitates cell adhesion, attachment and proliferation. It is most suitable for extrusion based bioprinting but due to the presence of high degrees of fibrous micro-architecture its use in inkjet bioprinter is limited. Other limitations of collagen based bio inks include, slow gelation rates, instability and formation of fibrous structure at high temperature [13]. A study has reported that cell attachment and cell proliferation of chondrocytes was enhanced by using collagen in conjunction with sodium alginate moreover it has also suppressed the differentiation of the incorporated cells and it is also suggested that the combination has also increased the mechanical strength of the structure thus it is preferred choice for the tissue engineering of the cartilage [14]. In another study has reported that viability and differentiation of human hepatocytes stem cells is improved when collagen was used in varying concentrations in lieu of alginate [15]. Engineered structures have shown enhanced biological and physical properties and a greater number of cells remained after printing. Moreover, engineered tissues and complex models of tumors were also developed by using the transglutaminase-crosslinked gelatin [16].

\section{Hyaluronic Acid Based Bionks}

Hyaluronic acid (HA) naturally occurs in all connective tissues and extra cellular matrix of cartilage of all mammals and shows the characteristics like collagen type I. HA is vastly used for tissue engineering as it is highly biocompatible and imparts flexibility to the hydrogels. Chemical modifications of HA increase its rheological properties and photo-cross linking. As HA plays a key role in embryonic development, thus it is favorable to the living cells in the hydrogels and structures bio printed using hyaluronic acid are more stable and mechanically controllable. However, owing to its slow gelation ability it is not suitable for extrusion based bioprinting, but it can be used efficiently by blending it with other hydrogels [1]. The adhesion property of hyaluronic acid based hydrogels was increased by the adding the oligopeptides having cell-adhesive properties while mechanical properties were not affected by this addition [17]. Moreover, Ruthenium-based complexes with visible light also polymerizes the hyaluronic acid-gelatin based bio inks. Owing to this ability cell differentiation and viability of adipose stem cells has been improved [18]. Additionally, hyaluronic acidcarboxymethyl cellulose increases the viability and stability of bioprinter structures, moreover the mechanical properties were also enhanced by modifying the HA based gels with methylcellulose [19].

\section{Fibrin-Based Bio inks}

Fibrin is the major clotting protein and can be transformed into hydrogel by the enzymatic reaction of thrombin and fibrinogen. It is highly biocompatible and possess biodegradation, but the structures formed using fibrin hydrogels are mechanically weak. It supports cell proliferation and growth. Due to its property of filaments formation the structures thus produced show high amount of deformation without breakage. It can be used with inkjet bioprinting, but it can cause nozzle blockage due to filament 
formation. In-vitro properties of fibrin has been investigated by developing a three dimensional structure of urethra [20]. Moreover, the combined effect of fibrin and hyaluronic acid by encapsulating the Schwan cells to investigate nerve regeneration [21].

\section{Matrigel TM}

Matrigel is a synthetic material and a commercial product mainly composed of gelatinous extra cellular material which promotes vascularization and outgrowth of cells from various tissue fragments, and promotes the differentiation of cultured cells, additionally complex cellular behavior is also observed when cells are cultured on Matrigel [22-23]. Matrigel imparts mechanical strength, stability and high survival rates to the 3D bioprinted structures as compared to alginate and agarose [24]. It is not a favorable choice as bio ink for extrusion based and droplet based bioprinters [25-26] but due to its optimal viscosity and thermal crosslinking ability it is widely used in laser based bioprinters [27]. Matrigel has been used as a vital instrument for the construction of rodent tumor xenograft models to develop innovative cancer treatments [28]. Moreover, Matrigel was used as a bio ink to print three dimensional biostructure using human osteosarcoma [29].

\section{Pluronic $®$ F-12}

Pluronic $\AA$ F-12 is a synthetic polymer having surfactant properties [30]. It is mostly used in combination with other bio ink materials e.g. PEG to deliver and slowed release of drugs [31] and with methacrylate hyaluronic acid to engineer biostructures with increased mechanical strength [32]. Complex structures can be engineered owing to the reversible properties of Pluronic $\AA$ F-12. Moreover perusable channel like structures can also be constructed using this material [33]. This material is acceptable for bioprinting for extrusion based bioprinter and but not favorable for droplet based bioprinting due to high viscosity and thermosensitive nature. A study reported that interaction ability of mesenchymal stem cells derived from bone marrow was increased when bioprinted with Pluronic $®$.

\section{Conclusion}

3D bioprinting technology is a futuristic approach for tissue engineering and synthesis of other biological structures with greater feasibility. By improving the quality of bio inks and scaling up the commercialization of 3D products can open new horizons. It can facilitate the patients who are in dire need of specific organs and dependent on the organ donor moreover it can also address the emergency medical needs of patients. Various bio inks with their advantage and disadvantages and their suitable bioprinters for the development of complex 3D biostructures are discussed in this review which will improve the commercial application of 3D printing technology. Natural and synthetic bio inks such as agarose and fibrin based inks and other extracellular matrix based bio inks are showing promising results but there is need to address the problems arising in the printing process. Moreover it is required to modify these bio inks so that single bio ink should have the capability to be used with various bioprinters. 3D bioprinting is a modern technology which has the potential to form such complex biostructures which will help to overcome the hurdles in all medical fields.

\section{References}

1. Janarthanan G, Insup N (2018) Recent trends in bioinks for 3D printing. Biomaterials Research 22: 11.

2. Kelly E (2018) FDA regulation of 3D-printed organs and associated ethical challenges. Univ PA Law Rev 166: 515-545.

3. Hölzl K, Lin S, Vlierberghe S, Gu L, Ovsianikov A (2016) Bioink properties before, during and after 3D bioprinting. Biofabrication 8(3): 032002.

4. Nakamura M, Kobayashi A, Takagi F, Watanabe A, Hiruma Y, et al. (2005) Biocompatible inkjet printing technique for designed seeding of individual living cells. Tissue Eng 11(11-12): 1658-1666.

5. Shor L, Güçeri S, Chang R, Gordon J, Kang Q, et al. (2009) Precision extruding deposition (PED) fabrication of polycaprolactone (PCL) scaffolds for bone tissue engineering. Biofabrication 1(1): 115003.

6. Fedorovich NE, Schuurman W, Wijnberg HM, Prins HJ, Van Weeren P R, et al. (2012) Biofabrication of osteochondral tissue equivalents by printing topologically defined, cell-laden hydrogel scaffolds. Tissue Eng 18(1): 33-44.

7. Guillotin B, Souquet A, Catros S, Duocastella M, Pippenger B, et al. (2010) Laser assisted bioprinting of engineered tissue with high cell density and microscale organization. Biomaterials. 31(28): 7250-7256.

8. Hospodiuk M, Dey M, Sosnoski D, Ozbolat IT (2017) The bioink: a comprehensive review on bioprintable materials. Biotechnol Adv 35(2): 217-239.

9. Serwer P, Allen JL, Hayes SJ (1983) Agarose gel electrophoresis of bacteriophages and related particles III. Dependence of gel sieving on the agarose preparation. Electrophoresis 4(3): 232-236.

10. Gu Q Tomaskovic Crook E, Kapsa R, Cook M, Zhou Q, et al. (2015) Bioprinting 3D functional neural tissue using human neural and induced pluripotent stem cells. InFront Bioeng Biotechnol. Conference Abstract: 10th World Biomaterials Congress.

11. Daly AC, Critchley SE, Rencsok EM, Kelly DJ (2016) A comparison of different bioinks for 3D bioprinting of fibrocartilage and hyaline cartilage. Biofabrication 8(4): 045002.

12. Hospodiuk M, Dey M, Sosnoski D, Ozbolat IT (2017) The Bioink: A Comprehensive Review on Bioprintable Materials. Biotechnology Advances 35(2): 217-239.

13. Ferreira AM, Gentile P, Chiono V, Ciardelli G (2012) Collagen for bone tissue regeneration. Acta Biomater 8(9): 3191-3200.

14. Yang X, Lu Z, Wu H, Li W, Zheng L, et al. (2018) Collagen-alginate as bioink for three-dimensional (3D) cell printing based cartilage tissue engineering. Mater Sci Eng C 83: 195-201.

15. Yeo M, Lee JS, Chun W, Kim GH (2016) An innovative collagen-based cell-printing method for obtaining human adipose stem cell-laden structures consisting of core-sheath structures for tissue engineering. Biomacromolecules. 17(4): 1365-1375.

16. Pimentel R, Ko SK, Caviglia C, Wolff A, Emnéus J, et al. (2018) Three dimensional fabrication of thick and densely populated soft constructs with complex and actively perfused channel network. Acta Biomater 65: 174-84.

17. Ouyang L, Highley CB, Rodell CB, Sun W, Burdick JA (2016) 3D printing of shearthinning hyaluronic acid hydrogels with secondary cross-linking. ACS Biomater Sci Eng 2(10): 1743-1751.

18. Sakai S, Ohi H, Hotta T, Kamei H, Taya M (2018) Differentiation potential of human adipose stem cells bioprinted with hyaluronic acid/gelatinbased bioink through microextrusion and visible light-initiated crosslinking. Biopolymers 109(2). 
19. Law N, Doney B, Glover H, Qin Y, Aman ZM, et al. (2018) Characterisation of hyaluronic acid methylcellulose hydrogels for 3D bioprinting. J. Mech Behav Biomed Mater 77: 389-399.

20. Zhang K, Fu Q, Yoo J, Chen X, Chandra P, et al. (2017) 3D bioprinting of urethra with PCL/PLCL blend and dual autologous cells in fibrin hydrogel: an in vitro evaluation of biomimetic mechanical property and cell growth environment. Acta Biomater 50: 154-164.

21. England S, Rajaram A, Schreyer DJ, Chen X (2017) Bioprinted fibrinfactor XIII hyaluronate hydrogel scaffolds with encapsulated Schwann cells and their in vitro characterization for use in nerve regeneration. Bioprinting 5: 1-9.

22. Kleinman HK, Martin GR (2005) Matrigel: Basement membrane matrix with biological activity. Semin. Cancer Biol 15(5): 378-386.

23. Gaetani R, Doevendans P, Metz CHG, Alblas J, Messina E, et al. (2012) Cardiac tissue engineering using tissue printing technology and human cardiac progenitor cells. Biomaterials 33(6): 1782-1790.

24. Melchels FPW, Domingos MN, Klein TJ, Malda J, Bartolo PJ, et al. (2012) Additive manufacturing of tissues and organs. Prog Polym Sci 37(8): 1079-1104.

25. Snyder JE, Hamid Q, Wang C, Chang R, Emami K, et al. (2011) Bioprinting cell-laden matrigel for radioprotection study of liver by pro-drug conversion in a dual-tissue microfluidic chip. Biofabrication 3(3): 034112.

26. Horváth L, Umehara Y, Jud C, Blank F, Petri Fink A, et al. (2015) Engineering an in vitro air blood barrier by 3D bioprinting Sci Rep 5: 7974 .

ISSN: 2574-1241

DOI: 10.26717/BJSTR.2018.11.002129

Saima Majeed. Biomed J Sci \& Tech Res

This work is licensed under Creative

Commons Attribution 4.0 License

Submission Link: https://biomedres.us/submit-manuscript.php
27. Schiele NR, Koppes RA, Corr DT, Ellison KS, Thompson DM, et al. (2009) Laser direct writing of combinatorial libraries of idealized cellular constructs: Biomedical applications. Appl Surf Sci 255(10): 5444-5447.

28. Benton G, Kleinman HK, George J, Arnaoutova I (2011) Multiple uses of basement membrane like matrix (BME/Matrigel) in vitro and in vivo with cancer cells. Int J Cancer 128(8): 1751-1757.

29. Barron JA, Spargo BJ, Ringeisen BR (2004) Biological laser printing of three dimensional cellular structures. Appl Phys A 79(4-6): 1027-1030.

30. Mesa M, Sierra L, Patarin J, Guth JL (2005) Morphology and porosity characteristics control of SBA-16 mesoporous silica. Effect of the triblock surfactant Pluronic F127 degradation during the synthesis. Solid State Sci 7(8): 990-997.

31. Gong CY, Shi S, Dong PW, Zheng XL, Fu SZ, et al. (2009) In vitro drug release behavior from a novel thermosensitive composite hydrogel based on Pluronic f127 and poly (ethylene glycol)-poly(epsilon-caprolactone)poly(ethylene glycol) copolymer. BMC Biotechnology 9: 8.

32. Müller M, Becher J, Schnabelrauch M, Zenobi Wong M (2015) Nanostructured Pluronic hydrogels as bioinks for 3D bioprinting. Biofabrication 7(3): 35006.

33. Wu W, De Coninck A, Lewis J (2011) Omnidirectional printing of 3D microvascular networks. Adv Mater. 23: H178-183.

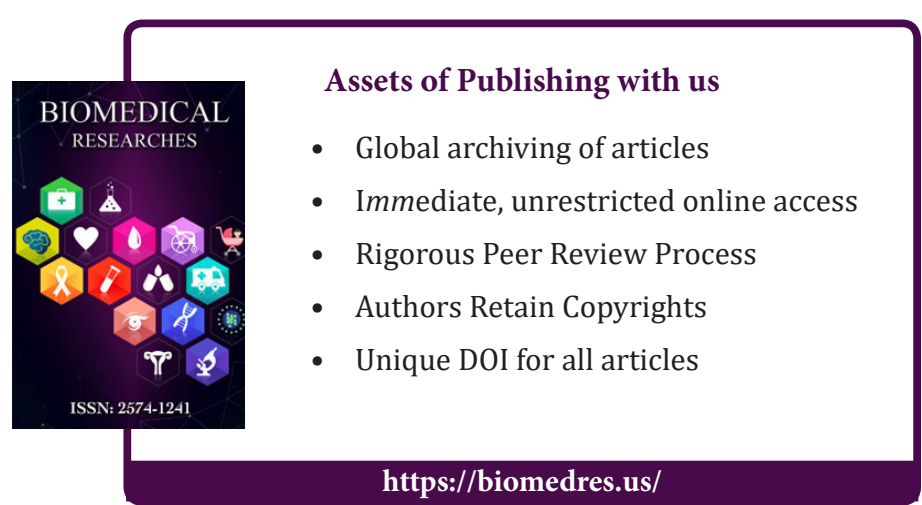

